\title{
Velocity Distribution of a Homogeneously Cooling Granular Gas
}

\author{
Peidong Yu $\odot,{ }^{1,2, *}$ Matthias Schröter $\odot,{ }^{1, \dagger}$ and Matthias Sperl@ ${ }^{1,2, \$}$ \\ ${ }^{1}$ Institut für Materialphysik im Weltraum, Deutsches Zentrum für Luft- und Raumfahrt (DLR), 51170 Cologne, Germany \\ ${ }^{2}$ Institut für Theoretische Physik, Universität zu Köln, 50937 Cologne, Germany
}

(Received 26 July 2019; revised manuscript received 27 November 2019; accepted 23 April 2020; published 22 May 2020)

\begin{abstract}
In contrast to molecular gases, granular gases are characterized by inelastic collisions and require therefore permanent driving to maintain a constant kinetic energy. The kinetic theory of granular gases describes how the average velocity of the particles decreases after the driving is shut off. Moreover, it predicts that the rescaled particle velocity distribution will approach a stationary state with overpopulated high-velocity tails as compared to the Maxwell-Boltzmann distribution. While this fundamental theoretical result was reproduced by numerical simulations, an experimental confirmation is still missing. Using a microgravity experiment that allows the spatially homogeneous excitation of spheres via magnetic fields, we confirm the theoretically predicted exponential decay of the tails of the velocity distribution.
\end{abstract}

DOI: 10.1103/PhysRevLett.124.208007

Introduction.-Granular gases consist of macroscopic particles, i.e., their diameter $d$ is micrometers or larger. From this property follow two important differences between granular and molecular gases. First, all collisions between granular particles are inelastic: a part of the mechanical energy of the relative motion of the particles is converted to heat [1]. Second, the energy scales involved in granular dynamics, such as the energy needed to lift a grain its own diameter, are 10 to 20 orders of magnitude larger than the thermal energy of the system. In consequence, maintaining a dynamic state of a granular gas does require the constant injection of energy.

However, these differences to molecular gases also signify the chance to "reinvent statistical mechanics in a new context" [2]. Properties of granular gases not known from their molecular counterpart include non-Fourier heat flow [3-5], correlations [6-8], breaking of time-reversal symmetry [9,10], segregation [11-18], nonequipartition [6,18-22], and clustering [23-32]. Sometimes granular gases unexpectedly behave even like equilibrium systems [33]. Motivated by these phenomena, theoretical physicists and mathematicians started, at the turn of the century, to extend the kinetic theory from nondissipative molecular systems [34] to dissipative particle systems; for recent summaries at the textbook level see Refs. [1,17].

The theoretical analysis of granular gases focuses on two stationary states: First the homogeneously driven gas where energy is injected in a spatially homogeneous way into the system in order to compensate the energy loss due to inelastic collisions. Kinetic theory predicts [35] that the distribution of individual particle velocities $P(v)$ develops overpopulated tails when compared to the Maxwell-Boltzmann distribution: $P(v) \sim \exp \left(-k v^{3 / 2}\right)$ for $v>\langle v\rangle / \epsilon$, where $\epsilon$ is the coefficient of restitution and $\langle v\rangle$ is the average velocity of all particles. Second, the homogeneous cooling state (HCS) where the system is not disturbed by external forces [35-40]. This results in a monotonous decrease of $\langle v\rangle$ with time $t$ according to the so-called Haff's law [41],

$$
\langle v(t)\rangle=v_{0} /(1+t / \tau)^{\gamma}
$$

$\tau$ is a material and density dependent characteristic timescale. If the coefficient of restitution $\epsilon$ of the particles can be considered to be velocity independent, $\gamma$ equals one. If the velocity dependence of $\epsilon$ for viscoelastic particles has to be taken into account, $\gamma$ becomes $5 / 6$.

The reason that the HCS is qualified as a stationary state, even though $\langle v\rangle$ is a function of time, is that the distribution of the rescaled particle velocities $c=v / v_{T}$, becomes stationary. $v_{T}$ is the velocity derived from granular temperature $T$ of the particles: $v_{T}=\sqrt{2 T / m}$ where $m$ is the particle mass. As discussed in Sec. 2 of the Supplemental Material [42], this thermal velocity can be computed as $v_{T}=2 / \sqrt{\pi}\langle v\rangle$. Kinetic theory [35,36] predicts that the tail of the velocity distribution $P(c)$ becomes exponential, i.e., $P(c) \sim \exp (-k c)$. The HCS ends with the onset of particle clustering which destroys its homogeneity [31].

Experimental confirmation of these kinetic theory results is difficult for two reasons. First, creating and maintaining a granular gas requires constant energy injection. Typically, this is done by vibrating the boundaries of the container, which, however, creates spatial and temporal inhomogeneities within the granular gas [25-28,47-50], thereby invalidating one of the preconditions of the kinetic theory approaches. A spatially homogeneous driving can be obtained by either restricting the experiment to a horizontally aligned two-dimensional system which is shaken vertically [51-55], or by using magnetic [30,56-59] or 
electrostatic [60] forces to drive a three-dimensional system in the bulk.

Second, on Earth the gravitational field will always collapse the granular gas into a granular solid where collisions are replaced by enduring contacts. This effect can again be counteracted by limiting the granular gas to a horizontally aligned two-dimensional system. This comes, however, at the price of a significantly larger number of particle-boundary rather than particle-particle collisions.

Force-free and three-dimensional granular gases can therefore only be realized by either levitating the particles in a magnetic field [30] or by performing the experiments in a real microgravity environment, as, e.g., realized in parabolic flights [28,50,53,61-63], drop towers [64,65], sounding rockets $[22,26,66]$, or satellites [67].

In consequence, experimental confirmations of the main results of the kinetic theory of granular gases have been scarce. A number of groups tried to measure the velocity distribution of the homogeneously driven gas using boundary-driven, two-dimensional [51-53,68-70] and threedimensional $[71,72]$ systems. They reported high energy tails proportional to $\exp \left(-k v^{\alpha}\right)$ with values of $\alpha$ in the range 0.8 to 2 . Especially for two-dimensional systems it has been shown that $\alpha$ is controlled by the friction coefficient of particle-sidewall collisions [73]. The only confirmations of $\alpha=1.5$ were obtained in an electrostatically driven three-dimensional system [60], and in a twodimensional, horizontal system containing rotationally driven disks [74].

For the homogeneous cooling state, the validity of Eq. (1) has been shown both in numerical simulations [31,75-77] and experiments [30,64,66]. For the exponents $\alpha$ characterizing the tail of the velocity distribution values in the range 0.6 to 1.5 have been reported for boundarydriven, two-dimensional systems [53]. The theoretically predicted exponential tail, i.e., $\alpha=1$, has up to now only been seen in numerical simulations [78,79]; an experimental confirmation is missing.

In this Letter, we describe a granular gas experiment which is both force-free, due to being performed in microgravity, and homogeneously heated via magnetic excitation. Our results confirm Haff's cooling law and show clear evidence of an exponential tail of the particle velocity distribution, thus directly confirming the validity of the kinetic theory of granular gases in the homogeneous cooling state.

Experimental setup.-The experiment was performed in a sounding rocket of the MAPHEUS campaign of German Aerospace Center (DLR) which provided 375 s of microgravity with a remnant gravity on the order of $10^{-5}$ times the earth acceleration [58]. The granular gas consists of 2796 spheres with a diameter $d=1.6 \pm 0.02 \mathrm{~mm}$. The particles are contained in a cubic container made of PMMA acrylic glass with an inner side length of $50 \mathrm{~mm}$. Taking into account the slanted corners of the cell,the average volume fraction of the gas is $\phi=0.05$ and the number density $n=0.0234 \mathrm{~mm}^{-3}$. The interior of the container is connected to the outside atmosphere, resulting in an air pressure $<0.01 \mathrm{~Pa}$ during the experiment. The resulting air drag reduces the particle velocity by $0.01 \%$ per second [58]

The spheres are made of ferro-magnetic MuMetall (Sekels $\mathrm{GmbH}$ ). Energy is injected into all granular particles in the bulk by pairs of magnets which are aligned along the diagonals of the cube, cf. Fig. 1. The excitation protocol cycles through the four pairs, switching each one on for $20 \mathrm{~ms}$ and then pausing for $80 \mathrm{~ms}$. The low coercivity of the particles ensures that the long-range interactions between the particles are negligible during cooling [58], while during heating, such long-range interactions actually contribute to a more isotropic excitation [59]. As shown in numerical simulations [59] and experiments [58], this type of excitation results in a homogeneously heated granular gas. It can also be seen in the movie of the experiment which is part of the Supplemental Material [42].

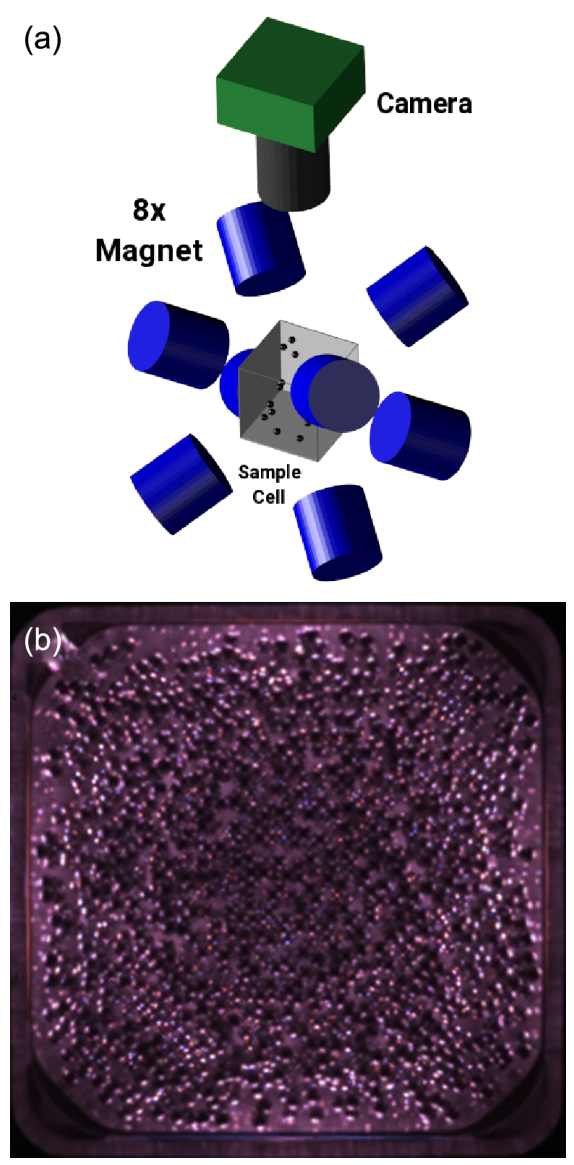

FIG. 1. Experimental setup. (a) The diagonals of a PMMA cube containing 2796 ferromagnetic spheres are aligned with four pairs of electromagnets that are used to inject energy into the granular gas. The time evolution of the granular gas is then observed with a camera at 165 frames/s. Panel (b) shows a typical example image. 
The granular dynamics inside the cell is observed with light field camera Raytrix R5 at 165 frames/s and a magnification of $70 \mu \mathrm{m} /$ pixel. Because of its reduced precision, the depth information provided by the light field camera is not used in our analysis. Section 1 of the Supplemental Material [42] provides further information on the two-dimensional image analysis.

Freely cooling granular gas.-During the flight of the sounding rocket four individual experiments were performed. Each experiment consisted of a first phase of at least $40 \mathrm{~s}$ duration during which the granular gas was heated by switching the magnetic fields on and off. Then the excitation was stopped and a cooling period of at least $40 \mathrm{~s}$ duration followed. However, as shown in Sec. 3 of the Supplemental Material [42], after about $4 \mathrm{~s}$ the remnant gravitation starts to induce spatial inhomogeneities in the sample cell. We therefore limit the subsequent analysis to the first $3 \mathrm{~s}$ of the cooling phase; these were sufficient to reach a stationary state as shown in Sec. 6 of the Supplemental Material [42].

Figure 2 presents the evolution of the average particle velocity in the last second of the heating and the first $3 \mathrm{~s}$ of the cooling phase. Two features of Fig. 2 are important to point out. First, Haff's law [Eq. (1), displayed in red] provides a good fit to all four experiments. We have tested both exponents $\gamma=1$ and 5/6; the differences are within our experimental uncertainty. This signifies that the velocity dependence of $\epsilon$ plays only a minor role in the velocity range analyzed here.

This is to be expected because for $\epsilon(g)=1-\zeta g^{1 / 5}+$ h.o.t. and within the studied range of relative collision velocities $g$, the change of epsilon is small ( $\zeta$ contains material parameters) [1]. We assume in our subsequent analysis a constant $\epsilon=0.66$, which is measured from lab calibrations (see Sec. 7 of Supplemental Material for details [42]).

Second, the reproducibility is high between the four experiments. The parameters $v_{0}$ and $\tau$ of the Haff fits agree within $\pm 8 \%$, and $v_{0} \tau$, which is determined by system properties alone, agrees within $\pm 3 \%$. Additionally, the particle velocities averaged over the last $2 \mathrm{~s}$ of the heating phase differ only by $\pm 0.3 \mathrm{~mm} / \mathrm{s}$ around the mean of the four experiments: $20.9 \mathrm{~mm} / \mathrm{s}$.

While the exact value of the fit parameter $v_{0}$ depends on the stochastic fluctuations within the granular gas during the heating phase, the value of $\tau$ can be compared to its prediction from granular kinetic theory $[1,35]$

$\tau_{\mathrm{k.t.}}=\left[\frac{\sqrt{2 \pi}}{3} \chi(\phi)\left(1+\frac{3}{16} a_{2}\right)\left(1-\epsilon^{2}\right) n d^{2} v_{T 0}\right]^{-1}$

Here, $\chi(\phi)$ is the contact value of the pair correlation function. We use the usual approximation for hard sphere systems [80]: $\chi(\phi)=(2-\phi) /\left[2(1-\phi)^{3}\right] . a_{2}$ is the second
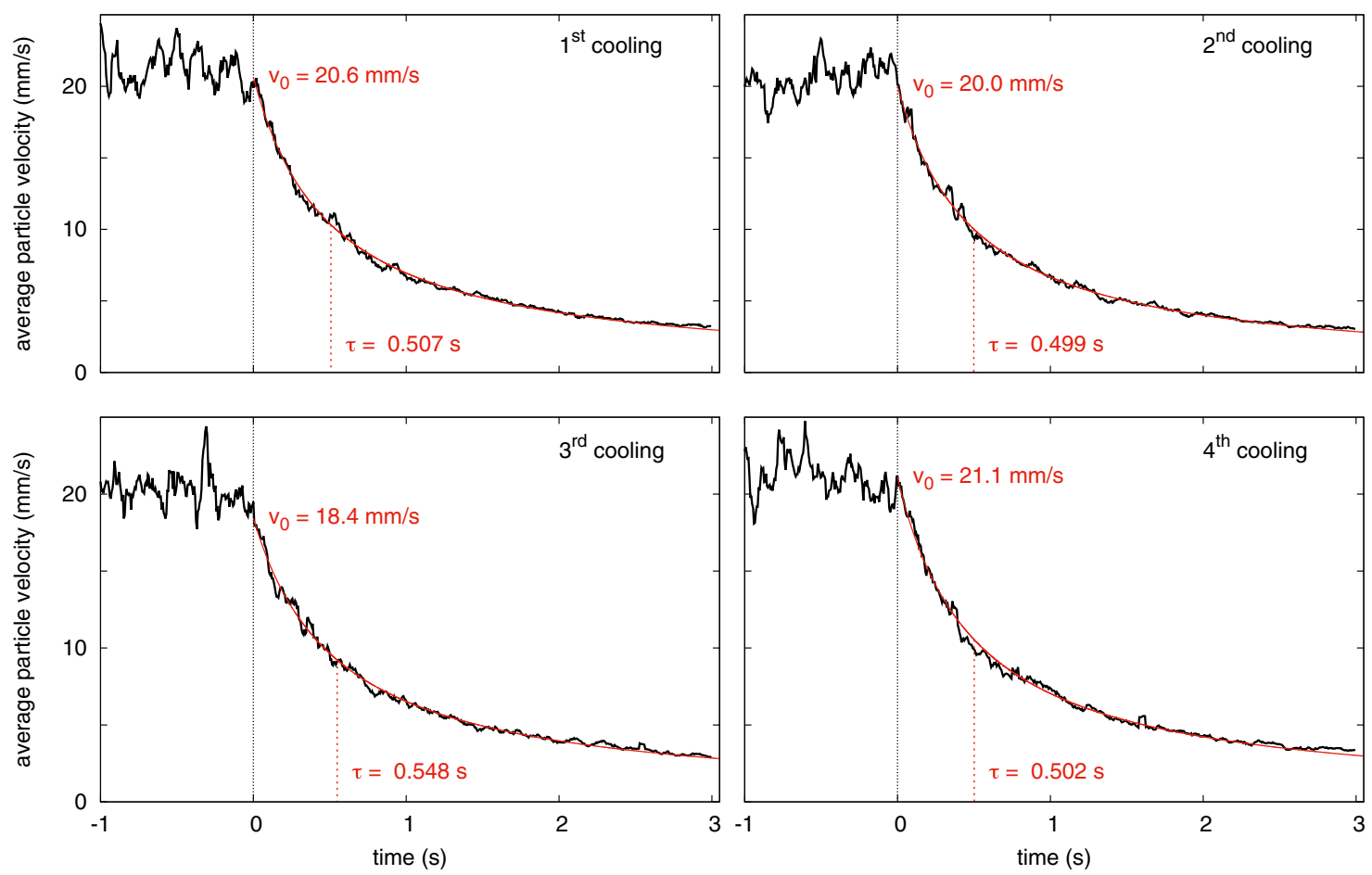

FIG. 2. All four experiments are well described by Haff's cooling law. Each panel displays first the last second of the magnetic driving with fluctuations in the average velocity. At $t=0$ the magnetic driving is switched off and the subsequent evolution of the average velocity is fit with Haff's law [Eq. (1)] assuming a constant coefficient of restitution (red line). Each fit provides two parameters: $v_{0}$, the average particle velocity at $t=0$, and $\tau$, the time by which the velocity has decreased to $v_{0} / 2$. 
Sonine coefficient: $a_{2}=16(1-\epsilon)\left(1-2 \epsilon^{2}\right) /[81-17 \epsilon+$ $\left.30 \epsilon^{2}(1-\epsilon)\right] . v_{T 0}$ is the thermal velocity of the particles at the beginning of the cooling: $v_{T 0}=2 / \sqrt{\pi} v_{0}$ (see Sec. 2 of the Supplemental Material for details [42]). Using the fitted value $v_{0}=20.6 \mathrm{~mm} / \mathrm{s}$ from our first experiment, Eq. (2) predicts $\tau_{\text {k.t. }}=1.34 \mathrm{~s}$, which is a factor of 2.6 times larger than the fitted value $\tau=0.507 \mathrm{~s}$.

The fact that $\tau<\tau_{\text {k.t. }}$ matches with the limits of Eq. (2) which includes only the energy dissipation in the normal direction of the collisions, and not dissipation in the tangential direction which can, e.g., be caused by interparticle friction. Furthermore, inclusion of tangential dissipation means the rotational degrees of freedom join the dissipation mechanism and couple with the translational motion [6]. We expect that theories for rough particles $[7,75,81,82]$ provide predictions closer to the experimental results. However, such a comparison would also require 3D particle tracking including the rotational motion of the particles. Therefore this question depicts the direction of future experimental improvements.

Velocity distribution.-Figure 3 shows the distribution of the rescaled particle velocities $P(c)$. The high reproducibility of the experiments allows us to improve the statistics by averaging the distributions of the four experiments; the four individual distributions can be found in Sec. 4 of the Supplemental Material [42]. For values of $c$ smaller than 1.5, $P(c)$ can be approximated by a Maxwell-Boltzmann distribution. However, the main result of Fig. 3 is that the high energy tail of $P(c)$ decays exponentially, as predicted by the kinetic theory of granular gases. A nonlinear fit of the experimental data using $P(c) \sim \exp \left(-k c^{\alpha}\right)$ results in $\alpha=0.96 \pm 0.19$, which is again in good agreement with kinetic theory.

Because of the intermittent nature of the magnetic driving (i.e., $20 \mathrm{~ms}$ on, $80 \mathrm{~ms}$ off), the velocity distribution

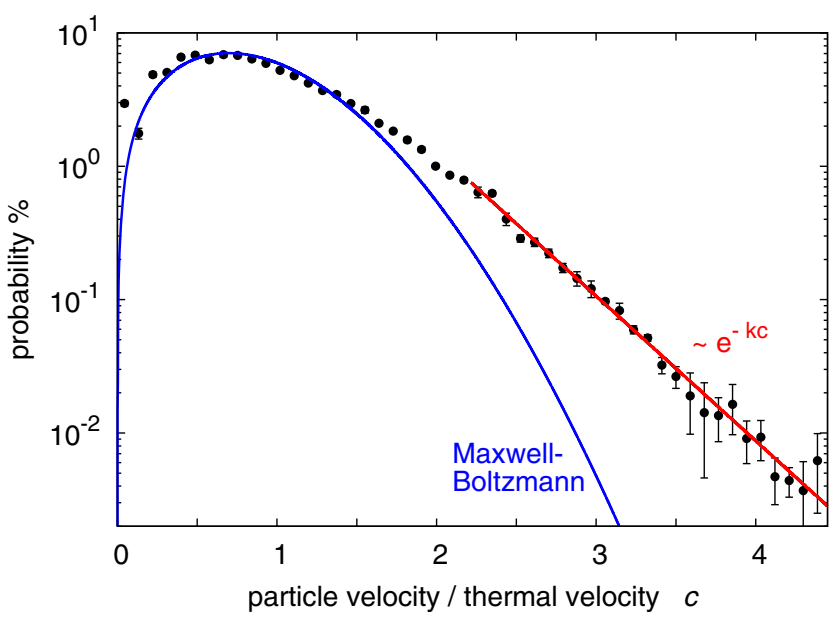

FIG. 3. The distribution of particle velocities in the homogeneous cooling state displays an exponential tail for $c>2.2$. Data are averaged over all four experiments shown in Fig. 2 and the time interval 1.8-3 s after onset of cooling. of the heated gas, which is shown in Sec. 5 of the Supplemental Material [42], is different from a MaxwellBoltzmann distribution and it does also not display a high velocity tail proportional to $\exp \left(-k v^{3 / 2}\right)$ as predicted by kinetic theory for the homogeneously driven gas. A fit of the tail with $P(c) \sim \exp \left(-k c^{\alpha}\right)$ results in $\alpha=0.72 \pm 0.21$.

The fact that the system still relaxes towards the distribution predicted by granular kinetic theory can be interpreted as the equivalent of Boltzmann's $H$ theorem for thermal systems [34]. It is essential for the comparison with theoretical predictions that the observed velocity histogram allows for the interpretation as a distribution function including the definition of a well-defined temperature.

Conclusions. - We have shown experimentally that the kinetic theory of granular gases predicts correctly the two most fundamental properties of a freely cooling threedimensional gas: the evolution of the average particle velocity and the appearance of an exponential tail in the particle velocity distribution. Building on this foundation, we expect quantitative deviations between experiment and existing theory to stimulate further fruitful work.

We acknowledge partial funding from BMWi/DLR through Projects No. 50WM1651 and 50WM1945. We also thank an anonymous referee for providing valuable insights into the theoretical implications of our data.

*peidong.yu@dlr.de

schroeter@science-consulting.info

*matthias.sperl@dlr.de

[1] N. Brilliantov and T. Pöschel, Kinetic Theory of Granular Gases (Oxford University Press, New York, 2004).

[2] L. P. Kadanoff, Built upon sand: Theoretical ideas inspired by granular flows, Rev. Mod. Phys. 71, 435 (1999).

[3] R. Soto, M. Mareschal, and D. Risso, Departure from Fourier's Law for Fluidized Granular Media, Phys. Rev. Lett. 83, 5003 (1999).

[4] J. W. Dufty, Fourier's law for a granular fluid, J. Phys. Chem. C 111, 15605 (2007).

[5] D. Candela and R. L. Walsworth, Understanding the breakdown of Fourier's law in granular fluids, Am. J. Phys. 75, 754 (2007).

[6] N. V. Brilliantov, T. Pöschel, W. T. Kranz, and A. Zippelius, Translations and Rotations are Correlated in Granular Gases, Phys. Rev. Lett. 98, 128001 (2007).

[7] W. Kranz, N. Brilliantov, T. Pöschel, and A. Zippelius, Correlation of spin and velocity in the homogeneous cooling state of a granular gas of rough particles, Eur. Phys. J. Special Topics 179, 91 (2009).

[8] B. Gayen and M. Alam, Effect of Coulomb friction on orientational correlation and velocity distribution functions in a sheared dilute granular gas, Phys. Rev. E 84, 021304 (2011).

[9] T. Pöschel, N. V. Brilliantov, and T. Schwager, Violation of molecular chaos in dissipative gases, Int. J. Mod. Phys. C 13, 1263 (2002). 
[10] R. S. Shaw, N. Packard, M. Schröter, and H. L. Swinney, Geometry-induced asymmetric diffusion, Proc. Natl. Acad. Sci. U.S.A. 104, 9580 (2007).

[11] S. S. Hsiau and M. L. Hunt, Granular thermal diffusion in flows of binary-sized mixtures, Acta Mech. 114, 121 (1996).

[12] J. T. Jenkins and D. K. Yoon, Segregation in Binary Mixtures under Gravity, Phys. Rev. Lett. 88, 194301 (2002).

[13] M. Schröter, S. Ulrich, J. Kreft, J. B. Swift, and H. L. Swinney, Mechanisms in the size segregation of a binary granular mixture, Phys. Rev. E 74, 011307 (2006).

[14] V. Garzó, Segregation in granular binary mixtures: thermal diffusion, Europhys. Lett. 75, 521 (2006).

[15] V. Garzó, Thermal diffusion segregation in granular binary mixtures described by the Enskog equation, New J. Phys. 13, 055020 (2011).

[16] J. J. Brey, N. Khalil, and J. W. Dufty, Thermal segregation beyond Navier-Stokes, New. J. Phys. 13, 055019 (2011).

[17] V. Garzó, Granular Gaseous Flows (Springer, Cham, 2019).

[18] D. Serero, C. Goldenberg, S. H. Noskowicz, and I. Goldhirsch, The classical granular temperature and slightly beyond, Powder Technol. 182, 257 (2008).

[19] A. Goldshtein and M. Shapiro, Mechanics of collisional motion of granular materials. Part 1. General hydrodynamic equations, J. Fluid Mech. 282, 75 (1995).

[20] K. Feitosa and N. Menon, Breakdown of Energy Equipartition in a 2D Binary Vibrated Granular Gas, Phys. Rev. Lett. 88, 198301 (2002).

[21] J. E. Galvin, S. R. Dahl, and C. M. Hrenya, On the role of Non-Equipartition in the dynamics of rapidly flowing granular mixtures, J. Fluid Mech. 528, 207 (2005).

[22] K. Harth, U. Kornek, T. Trittel, U. Strachauer, S. Höme, K. Will, and R. Stannarius, Granular Gases of Rod-Shaped Grains in Microgravity, Phys. Rev. Lett. 110, 144102 (2013).

[23] I. Goldhirsch and G. Zanetti, Clustering Instability in Dissipative Gases, Phys. Rev. Lett. 70, 1619 (1993).

[24] I. Goldhirsch, M.-L. Tan, and G. Zanetti, A molecular dynamical study of granular fluids $\mathrm{i}$ : The unforced granular gas in two dimensions, J. Sci. Comput. 8, 1 (1993).

[25] A. Kudrolli, M. Wolpert, and J. P. Gollub, Cluster Formation due to Collisions in Granular Material, Phys. Rev. Lett. 78, 1383 (1997).

[26] E. Falcon, R. Wunenburger, P. Evesque, S. Fauve, C. Chabot, Y. Garrabos, and D. Beysens, Cluster Formation in a Granular Medium Fluidized by Vibrations in Low Gravity, Phys. Rev. Lett. 83, 440 (1999).

[27] E. Opsomer, F. Ludewig, and N. Vandewalle, Phase transitions in vibrated granular systems in microgravity, Phys. Rev. E 84, 051306 (2011).

[28] M. Noirhomme, A. Cazaubiel, A. Darras, E. Falcon, D. Fischer, Y. Garrabos, C. Lecoutre-Chabot, S. Merminod, E. Opsomer, F. Palencia, J. Schockmel, R. Stannarius, and N. Vandewalle, Threshold of gas-like to clustering transition in driven granular media in low-gravity environment, Europhys. Lett. 123, 14003 (2018).

[29] P. P. Mitrano, V. Garzó, A. M. Hilger, C. J. Ewasko, and C. M. Hrenya, Assessing a hydrodynamic description for instabilities in highly dissipative, freely cooling granular gases, Phys. Rev. E 85, 041303 (2012).
[30] C. C. Maaß, N. Isert, G. Maret, and C. M. Aegerter, Experimental Investigation of the Freely Cooling Granular Gas, Phys. Rev. Lett. 100, 248001 (2008).

[31] M. Hummel, J. P. D. Clewett, and M. G. Mazza, A universal scaling law for the evolution of granular gases, Europhys. Lett. 114, 10002 (2016).

[32] E. Opsomer, M. Noirhomme, N. Vandewalle, E. Falcon, and S. Merminod, Segregation and pattern formation in dilute granular media under microgravity conditions, npj Microgravity 3, 1 (2017).

[33] K. Nichol and K. E. Daniels, Equipartition of Rotational and Translational Energy in a Dense Granular Gas, Phys. Rev. Lett. 108, 018001 (2012).

[34] S. Chapman and T. G. Cowling, The Mathematical Theory of Nonuniform Gases (Cambridge University Press, Cambridge, England, 1960).

[35] T. Van Noije and M. Ernst, Velocity distributions in homogeneous granular fluids: the free and the heated case, Granular Matter 1, 57 (1998).

[36] S. E. Esipov and T. Pöschel, The granular phase diagram, J. Stat. Phys. 86, 1385 (1997).

[37] A. Puglisi, V. Loreto, U. Marini Bettolo Marconi, and A. Vulpiani, Kinetic approach to granular gases, Phys. Rev. E 59, 5582 (1999).

[38] I. Goldhirsch, S. H. Noskowicz, and O. Bar-Lev, The homogeneous cooling state revisited, in Granular Gas Dynamics, Lecture Notes in Physics, edited by T. Pöschel and N. Brilliantov (Springer Berlin Heidelberg, Berlin, Heidelberg, 2003), pp. 37-63.

[39] T. Pöschel, N. V. Brilliantov, and A. Formella, Impact of high-energy tails on granular gas properties, Phys. Rev. E 74, 041302 (2006).

[40] T. Pöschel, N. V. Brilliantov, and A. Formella, Granular gas cooling and relaxation to the steady state in regard to the over populated tail of thevelocity distribution, Int. J. Mod. Phys. C 18, 701 (2007).

[41] P. K. Haff, Grain flow as a fluid-mechanical phenomenon, J. Fluid Mech. 134, 401 (1983).

[42] See Supplemental Material at http://link.aps.org/ supplemental/10.1103/PhysRevLett.124.208007 for the image processing methods, further elaboration of theories, additional details of statistical analysis, and the descriptions of the calibration experiment, which includes Refs. [1,40,43-46]. See also movie of particle dynamics several seconds before and after the start of the cooling.

[43] R. Besseling, L. Isa, E. R. Weeks, and W. C. Poon, A quantitative study of three-dimensional Lagrangian particle tracking algorithms, Adv. Colloid Interface Sci. 146, 1 (2009).

[44] C. Dalitz, T. Schramke, and M. Jeltsch, Quantitative imaging of colloidal flows, Image Process. Line 7, 184 (2017).

[45] K. Jaqaman, D. Loerke, M. Mettlen, H. Kuwata, S. Grinstein, S. L. Schmid, and G. Danuser, Iterative Hough Transform for line detection in 3D point vlouds, Nat. Methods 5, 695 (2008).

[46] N. T. Ouellette, H. Xu, and E. Bodenschatz, Robust singleparticle tracking in live-cell time-lapse sequences, Exp. Fluids 40, 301 (2006). 
[47] J. Bougie, Sung Joon Moon, J. B. Swift, and H. L. Swinney, Shocks in vertically oscillated granular layers, Phys. Rev. E 66, 051301 (2002).

[48] B. Meerson, T. Pöschel, P. V. Sasorov, and T. Schwager, Giant fluctuations at a granular phase separation threshold, Phys. Rev. E 69, 021302 (2004).

[49] P. Eshuis, K. van der Weele, D. van der Meer, R. Bos, and D. Lohse, Phase diagram of vertically shaken granular matter, Phys. Fluids 19, 123301 (2007).

[50] A. Sack, M. Heckel, J.E. Kollmer, F. Zimber, and T. Pöschel, Energy Dissipation in Driven Granular Matter in the Absence of Gravity, Phys. Rev. Lett. 111, 018001 (2013).

[51] J.S. Olafsen and J.S. Urbach, Clustering, Order, and Collapse in a Driven Granular Monolayer, Phys. Rev. Lett. 81, 4369 (1998).

[52] G. W. Baxter and J. S. Olafsen, Kinetics: Gaussian statistics in granular gases, Nature (London) 425, 680 (2003).

[53] S. Tatsumi, Y. Murayama, H. Hayakawa, and M. Sano, Experimental study on the kinetics of granular gases under microgravity, J. Fluid Mech. 641, 521 (2009).

[54] P. M. Reis, R. A. Ingale, and M. D. Shattuck, Crystallization of a Quasi-Two-Dimensional Granular Fluid, Phys. Rev. Lett. 96, 258001 (2006).

[55] J. Deseigne, O. Dauchot, and H. Chaté, Collective Motion of Vibrated Polar Disks, Phys. Rev. Lett. 105, 098001 (2010).

[56] M. Schmick and M. Markus, Gaussian distributions of rotational velocities in a granular medium, Phys. Rev. E 78, 010302(R) (2008).

[57] E. Falcon, J.-C. Bacri, and C. Laroche, Equation of state of a granular gas homogeneously driven by particle rotations, Europhys. Lett. 103, 64004 (2013).

[58] P. Yu, E. Stärk, G. Blochberger, M. Kaplik, M. Offermann, D. Tran, M. Adachi, and M. Sperl, Magnetically excited granular matter in low gravity, Rev. Sci. Instrum. 90, 054501 (2019).

[59] M. Adachi, P. Yu, and M. Sperl, Magnetic excitation of a granular gas as a bulk thermostat, npj Microgravity 5, 19 (2019).

[60] I. S. Aranson and J. S. Olafsen, Velocity fluctuations in electrostatically driven granular media, Phys. Rev. E 66, 061302 (2002).

[61] E. Falcon, S. Aumaître, P. Evesque, F. Palencia, C. Lecoutre-Chabot, S. Fauve, D. Beysens, and Y. Garrabos, Collision statistics in a dilute granular gas fluidized by vibrations in low gravity, Europhys. Lett. 74, 830 (2006).

[62] M. Leconte, Y. Garrabos, E. Falcon, C. Lecoutre-Chabot, F. Palencia, P. Évesque, and D. Beysens, Microgravity experiments on vibrated granular gases in a dilute regime: non-classical statistics,J. Stat. Mech. (2006) P07012.

[63] Y. Grasselli, G. Bossis, and R. Morini, Translational and rotational temperatures of a $2 \mathrm{~d}$ vibrated granular gas in microgravity, Eur. Phys. J. E 38, 8 (2015).

[64] D. Heielmann, J. Blum, H. J. Fraser, and K. Wolling, Microgravity experiments on the collisional behavior of saturnian ring particles, Icarus 206, 424 (2010).
[65] P. Born, J. Schmitz, and M. Sperl, Dense fluidized granular media in microgravity, npj Microgravity 3, 27 (2017).

[66] K. Harth, T. Trittel, S. Wegner, and R. Stannarius, Free Cooling of a Granular Gas of Rod-Like Particles in Microgravity, Phys. Rev. Lett. 120, 214301 (2018).

[67] M. Hou, R. Liu, G. Zhai, Z. Sun, K. Lu, Y. Garrabos, and P. Evesque, Velocity distribution of vibration-driven granular gas in Knudsen regime in microgravity, Microgravity Sci. Technol. 20, 73 (2008).

[68] A. Kudrolli and J. Henry, Non-Gaussian velocity distributions in excited granular matter in the absence of clustering, Phys. Rev. E 62, R1489 (2000).

[69] F. Rouyer and N. Menon, Velocity Fluctuations in a Homogeneous 2D Granular Gas in Steady State, Phys. Rev. Lett. 85, 3676 (2000).

[70] D. L. Blair and A. Kudrolli, Collision statistics of driven granular materials, Phys. Rev. E 67, 041301 (2003).

[71] W. Losert, D. G. W. Cooper, J. Delour, A. Kudrolli, and J. P. Gollub, Velocity statistics in excited granular media, Chaos 9, 682 (1999).

[72] C. Huan, X. Yang, D. Candela, R. W. Mair, and R. L. Walsworth, NMR experiments on a three-dimensional vibrofluidized granular medium, Phys. Rev. E 69, 041302 (2004).

[73] J. S. van Zon, J. Kreft, D. I. Goldman, D. Miracle, J. B. Swift, and H.L. Swinney, Crucial role of side-walls in velocity distributions in quasi-two-dimensional granular gases, Phys. Rev. E 70, 040301(R) (2004).

[74] C. Scholz and T. Pöschel, Velocity Distribution of a Homogeneously Driven Two-Dimensional Granular Gas, Phys. Rev. Lett. 118, 198003 (2017).

[75] S. Luding, M. Huthmann, S. McNamara, and A. Zippelius, Homogeneous cooling of rough, dissipative particles: theory and simulations, Phys. Rev. E 58, 3416 (1998).

[76] T. Kanzaki, R. C. Hidalgo, D. Maza, and I. Pagonabarraga, Cooling dynamics of a granular gas of elongated particles, J. Stat. Mech. (2010) P06020.

[77] A. Bodrova, A. V. Chechkin, A. G. Cherstvy, and R. Metzler, Quantifying non-ergodic dynamics of force-free granular gases, Phys. Chem. Chem. Phys. 17, 21791 (2015).

[78] J. J. Brey and M. J. Ruiz-Montero, Direct Monte Carlo simulation of dilute granular flow, Comput. Phys. Commun. 121-122, 278 (1999).

[79] J. J. Brey, D. Cubero, and M. J. Ruiz-Montero, High energy tail in the velocity distribution of a granular gas, Phys. Rev. E 59, 1256 (1999).

[80] N. F. Carnahan and K. E. Starling, Equation of state for nonattracting rigid spheres, J. Chem. Phys. 51, 635 (1969).

[81] A. Santos, G. M. Kremer, and M. dos Santos, Sonine approximation for collisional moments of granular gases of inelastic rough spheres, Phys. Fluids 23, 030604 (2011).

[82] A. Bodrova and N. Brilliantov, Self-diffusion in granular gases: an impact of particles roughness, Granular Matter 14, 85 (2012). 